\title{
Prevalence and factors associated with nontuberculous mycobacteria in non-cystic fibrosis bronchiectasis: a multicenter observational study
}

\author{
L. Máiz ${ }^{1 *}$, R. Girón ${ }^{2}$, C. Olveira ${ }^{3}$, M. Vendrell ${ }^{4}$, R. Nieto ${ }^{1}$ and M. A. Martínez-García ${ }^{5}$
}

\begin{abstract}
Background: Data on the prevalence of and factors associated with nontuberculous mycobacteria (NTM) in patients with non-cystic fibrosis (CF) bronchiectasis are limited. Our aim was to determine the prevalence and factors associated with isolation of NTM in this population.

Methods: We performed a multicenter observational study of historical cohorts comprising consecutive patients with non-CF bronchiectasis and at least 2 sputum samples cultured for mycobacteria over a period of 5 years.

Results: The study population included 218 adult patients (61.9\% women) with a mean (SD) age of 55.7 (16) years and a mean (SD) of 5.1 (3.3) cultures/patient. NTM was isolated from sputum in 18 patients (8.3 \%). Of these, 5 patients (28\%) met the American Thoracic Society criteria for NTM disease. Mycobacterium avium complex was the most frequently isolated microorganism (9 patients, $4.1 \%$ ). The variables independently associated with isolation of NTM were FVC $\geq 75 \%$ predicted $(\mathrm{OR}, 4.84 ; 95 \% \mathrm{Cl} 1.47$ to $15.9 ; p<0.05)$, age $\geq 50$ years $(\mathrm{OR}, 4.74 ; 95 \% \mathrm{Cl} 1.25$ to 17.97; $p<0.05)$, and body mass index (BMI) $\leq 23 \mathrm{~kg} / \mathrm{m}^{2}(\mathrm{OR}, 2.97 ; 95 \% \mathrm{Cl} 1.03-8.58 ; p<0.05)$. Patients with these three characteristics had a $40 \%$ probability of having at least one isolation of NMT.

Conclusions: A significant number of patients with non-CF bronchiectasis are positive for the isolation of NTM. M. avium complex is the most frequently isolated mycobacteria. FVC $\geq 75 \%$ predicted, age $\geq 50$ years, and a BMI $\leq$ $23 \mathrm{~kg} / \mathrm{m}^{2}$ were independently associated with the presence of NTM in patients with non-CF bronchiectasis.
\end{abstract}

Keywords: Nontuberculous mycobacteria, Disease prevalence, Non-cystic fibrosis bronchiectasis, Epidemiology Abbreviations: BMI, Body mass index; CF, Cystic fibrosis; NTM, Nontuberculous mycobacteria; PPMs, Potential pathogenic microorganisms

\section{Background}

Bronchiectasis is a chronic respiratory condition characterized by cough, sputum production, and increased susceptibility to lower respiratory tract infections. Impairment of mucus clearance facilitates recurrent respiratory infections [1]. While the lower respiratory tract is normally sterile, conditions such as bronchiectasis enable colonization by microbes such as bacteria. However, the airways of patients

\footnotetext{
*Correspondence: luis.maiz@salud.madrid.org

${ }^{1}$ Pneumology Service, Chronic Bronchial Infection, Cystic Fibrosis and Bronchiectasis Unit, Hospital Universitario Ramón y Cajal, Carretera de Colmenar Km 9,100, 28034 Madrid, Spain

Full list of author information is available at the end of the article
}

with bronchiectasis are also chronically colonized or infected by microorganisms, such as yeasts, filamentous fungi, and nontuberculous mycobacteria (NTM) [2]. NTM are ubiquitous in water and soil. Most cases of human infection are caused by NTM from the environment [3].

The incidence of lung colonization and infection due to NTM in patients with cystic fibrosis (CF) and non-CF bronchiectasis is increasing worldwide [4]. Since NTM diseases are not notifiable, epidemiological data are not readily available. The organism most frequently associated with these infections is Mycobacterium avium complex [5-7]. Isolation of pulmonary NTM does not necessarily indicate advanced infection or "disease," since NTM may 
be present in respiratory tract secretions with no evident signs of illness ("colonization"). In pre-existing lung disease, especially in patients who experience frequent exacerbations (eg, those with bronchiectasis), defining clinical and radiological criteria that are specific for NTM diseases is more difficult.

Most published data on the prevalence of and factors associated with NTM in bronchiectasis are obtained from patients with CF. A recent meta-analysis by Chu et al. showed that the overall prevalence of NTM was $9.3 \%$ in patients with non-CF bronchiectasis. Nevertheless, data on patients with non-CF bronchiectasis are limited, and most studies in this population have small samples [7]. Therefore, the aims of the present study were to determine the prevalence of NTM in a multicentre cohort of consecutive adult patients with non-CF bronchiectasis and to determine factors that are independently associated with isolation of NTM.

\section{Methods}

\section{Study design}

We performed an observational study of historical cohorts from 4 Spanish teaching hospitals with multidisciplinary and standardized non-CF bronchiectasis outpatient clinics.

\section{Study population}

The study population comprised 296 consecutive patients aged $\geq 18$ years who had been diagnosed with non-CF bronchiectasis of widely varying causes and for whom radiological extension and clinical and functional impairment were confirmed. Patients had to have been followed for at least 5 years during the period 2002-2010 before they could be considered for inclusion in analysis.

Patients had to have at least 2 sputum samples cultured for mycobacteria while (in a clinically stable phase) during the 5 years after the diagnosis. According to the recommendations of the Spanish Society of Pulmonology and Thoracic Surgery, the causes ruled out in idiopathic bronchiectasis were as follows: immunodeficiency with evidence of defective antibody production, gastroesophageal reflux disease, allergic bronchopulmonary aspergillosis, mycobacterial infections prior to development or diagnosis of bronchiectasis, cystic fibrosis, primary ciliary dyskinesia, and $\alpha 1$-antitrypsin deficiency [8]. CF was ruled out by 2 negative sweat test results in patients with bronchiectasis of unknown cause or a clinical presentation compatible with CF [9]. The study was approved by the Ethics and Research Committee of each center (registration number of the coordinating center: 008889-2011).

\section{Diagnosis of bronchiectasis}

Bronchiectasis was diagnosed based on a high-resolution computed tomography scan of the chest that was interpreted by radiologists experienced in respiratory disorders. Images were obtained in full inspiration (1-mm collimation and $10-\mathrm{mm}$ intervals from the apex to the base of the lungs). The presence of bronchiectasis was confirmed based on the criteria published by Naidich et al. [10]. The extent of bronchiectasis was evaluated according to the number of lobes affected, with the lingula and middle lobe considered as independent lobes.

\section{Data collection}

Data were collected from all clinically stable patients and included the following: age, gender, body mass index (BMI, $\mathrm{kg} / \mathrm{m}^{2}$ ), etiology, smoking habit (pack-years), dyspnea according to the modified Medical Research Council scale, macroscopic appearance of sputum (percentage of patients with purulent sputum), type of bronchiectasis (cystic or noncystic), radiological findings (number of lobes affected by bronchiectasis), and spirometry findings (forced vital capacity [FVC] and forced expiratory volume in the first second $\left[\mathrm{FEV}_{1}\right]$ as both absolute values and percent predicted). We also recorded hospitalizations secondary to severe exacerbations and the number of exacerbations. All variables were obtained within 6 months after the radiological diagnosis of bronchiectasis, except for hospitalizations and the number of exacerbations, which were obtained prospectively during the year after the radiological diagnosis. Long-term treatments (antibiotics, oral macrolides, and oral corticosteroids) taken for at least 1 year after the radiological diagnosis of bronchiectasis were also recorded.

One sputum sample was recovered every 6 months during a clinically stable phase and cultured for mycobacteria, bacteria, and fungi. Additional sputum cultures were obtained whenever considered necessary by the clinician.

A stable clinical situation was defined as the absence of clinical criteria of exacerbation and no antibiotics or corticosteroids in the preceding 4 weeks [11]. Exacerbation was defined as the acute onset and persistence of changes in sputum characteristics (increased volume, thicker consistency, greater purulence, and hemoptysis) and/or increased breathlessness unrelated to other causes [12].

\section{Microbiology}

Each respiratory sample was collected under sterile conditions and processed immediately or conserved at $4{ }^{\circ} \mathrm{C}$. Smears were stained using the auramine acid fast method and scanned with microscopy fluorescence light at $\times 200$ and $\times 400$. All respiratory samples were processed according to the Tacquet-Tison technique. 
Processed samples were inoculated on LowensteinJensen, Coletsos, and liquid medium (VersaTREK, formerly ESPII, Difco, Detroit, Michigan, USA). Cultures were incubated at $37{ }^{\circ} \mathrm{C}$ for at least 8 weeks.

Classic phenotyping assays were used. A specific gene probe for detection of $M$. avium complex was first used in 1990 (Accuprobe, GenProbe Inc., San Diego, California, USA). In 2003, species identification was complemented by $16 \mathrm{~S}$ rRNA gene sequence analysis.

Sputum samples underwent auramine acid-fast staining and examination for routine bacteriological examination simultaneously. Only sputum samples with more than 25 polymorphonuclear leukocytes and fewer than 10 squamous cells on Gram stain were considered valid samples and processed for bacterial culture. Bacterial chronic lung infection was defined as isolation of the same potentially pathogenic microorganism (PPM) after the diagnosis of bronchiectasis in $>50 \%$ of respiratory cultures during the 5-year study period [13].

\section{Statistical analysis}

The statistical analysis was performed using SPSS, version 15.0 (SPSS, Chicago, Illinois, USA). All data were expressed as mean (SD) or median (IQR) for quantitative variables and as absolute values and percentages for qualitative variables. The normality of the distribution was assessed using the Kolmogorov-Smirnov test. Prevalence of isolation of NTM was defined as the percentage of patients with at least 1 positive culture for NTM during the study. Patients were divided into 2 groups: NTM-positive patients (those with at least 1 positive sputum culture that showed growth of mycobacteria at any visit) and NTM-negative patients. Depending on the distribution of the variables, the $t$ test or Mann-Whitney test was used to compare 2 means, and the chi-square test (with Fisher exact test if necessary) was used to compare qualitative or dichotomous variables. Variables of clinical interest (according to the current literature or the researcher's opinion) and those that presented statistically significant differences $(p<0.1)$ in the univariate analysis were included as independent variables in a logistic regression model based on the backward stepwise technique (Wald test). In order to determine the probability of having at least 1 isolation of NTM in different patient profiles, ROC curves were used to dichotomize continuous variables of special clinical interest according to the researchers (age, FVC, and BMI). The cut-off points were those with the greatest capacity to discriminate between patients with and without NTM, as follows: FVC (\%predicted, with a cut-off of $75 \%$ ), age (with a cut-off of 50 years), and BMI (with a cut-off point of $23 \mathrm{~kg} / \mathrm{m}^{2}$ ).

The independent variables that were finally included were age $\geq 50$ years, $\mathrm{BMI} \leq 23 \mathrm{~kg} / \mathrm{m}^{2}$, macroscopic appearance of the sputum (mucoid vs mucopurulent or purulent), FVC $\geq 75 \%$, and isolation of PPMs (apart from NTM) from the sputum. The odds ratio (OR) and confidence intervals $(95 \% \mathrm{CI})$ for the independent variables were also calculated. $P$ values $<0.05$ were considered statistically significant in the logistic regression analysis.

A figure was constructed to show the different patient profiles with their probabilities of having at least 1 isolation of NTM depending of the presence or absence of the dichotomized variables independently associated with the isolation of NTM.

\section{Results}

\section{Study population}

Of the 296 patients screened, 218 met the eligibility criteria and were included in the study. The mean (SD) age of patients was 55.7 (16) years and $61.9 \%$ were women. FVC was $66.8 \pm 21.6 \%$ predicted, $\mathrm{FEV}_{1} 62.6 \pm$ $26.1 \%$ predicted, and BMI $24.8(4.6) \mathrm{kg} / \mathrm{m}^{2}$. All patients had at least 2 sputum samples cultured for mycobacteria during the 5-year study, with a mean of 5.1 (3.3) cultures/patient. The most frequent known aetiology of bronchiectasis was postinfectious causes (35.8\%), followed by chronic obstructive pulmonary disease (13.8\%), systemic diseases (12.8\%), immunodeficiency $(2.3 \%)$, and primary ciliary dyskinesia $(3.2 \%)$. The aetiology was unknown in $29.8 \%$ of cases. Cystic bronchiectasis was detected in 56 patients (25.7\%).

\section{Prevalence of mycobacterial species}

NTM was isolated at least once from sputum in 18 patients $(8.3 \%)$ and $M$. tuberculosis from 2 patients. $M$. avium complex was the most frequently isolated microorganism (9 patients, $4.1 \%)$. Other mycobacterial species were recovered from 9 patients $(4.1 \%)$ and included M. abscessus (3 patients), M. fortuitum (2), M. gordonae (2), M. chelonae (1), M. lentiflavum (1), and M. simiae (1). One patient had M. simiae and M. avium complex. Smears were positive in 7 of the 218 eligible patients (3.2\%). Of the 18 patients in whom NTM was isolated, only $6(33 \%)$ were smear-positive, and 5 (28\%) met the ATS/IDSA criteria for NTM lung disease [14] (3 $M$. avium complex, 1 M. abscessus, $1 M$. chelonae) (Fig. 1).

\section{Cohort characteristics and factors associated with isolation and disease caused by NTM}

When we compared the 5 patients who met the ATS/ IDSA criteria for NTM lung disease with the remaining patients (213), we found that the 5 patients were women. With respect to the remainder of the study population, these 5 patients were less likely to present cystic bronchiectasis ( 0 vs $26 \%, p=0.001$ ) or chronic bacterial colonization (20\% vs $59 \% ; p=0.01)$ and had better 


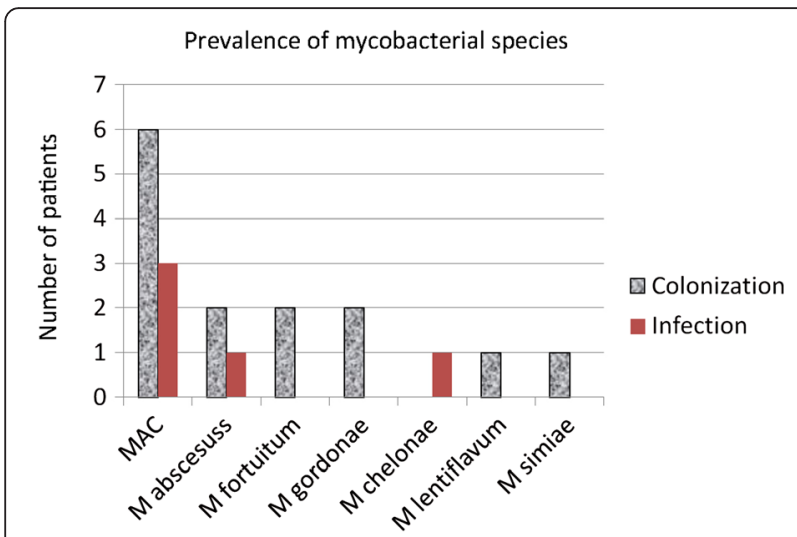

Fig. 1 Number of patients with positive non-tuberculous mycobacterial cultures (NTM), grouped by mycobacterial species. Patients who met American Thoracic Society/Infectious Disease Society of America (ATS/ IDSA) microbiologic criteria for NTM disease are represented in the shaded bars and those who did not meet ATS/IDSA criteria are represented in solid bars. The patient with Mycobacterium simiae also had M. avium complex (MAC)

functional test results (FEV1 $80.5 \%$ vs $62.1 \%$ [ $p=0.035]$ and FVC de $85 \%$ vs $74 \%(p=0.0429)$. None of the 5 patients died during follow-up.

The characteristics of patients in the NTM-positive and NTM-negative groups are shown in Table 1. Univariate analysis revealed that patients with NTM were significantly older (64 vs 54.9 years; $p<0.05$ ) and tended to be less frequently chronically infected with Pseudomonas aeruginosa (28 \% vs. $44 \%)$, Haemophilus influenzae (11\% vs. $23 \%)$, and other PPMs (12\% vs. $45 \%)$. In addition, Aspergillus was more frequently isolated than in culture-negative patients (39\% vs. $26 \%$ ), purulent sputum was less frequent ( $41 \%$ vs. $62 \%$ ), and lung function was better.

Table 2 shows the results of the fully adjusted logistic regression analysis. Patients with isolation of NTM were nearly 5 times as likely to be aged $\geq 50$ years and have an $\mathrm{FVC}>75 \%$ predicted value and nearly 3 times as likely to have a $\mathrm{BMI} \leq 23 \mathrm{~kg} / \mathrm{m}^{2}$.

Figure 2 shows a patient profile probability tree for the isolation of NTM at least once depending on the 3 variables independently related to isolation of NTM. The patient profile with the greatest probability $(40 \%)$ of isolation of NTM comprised all 3 characteristics (FVC predicted $\geq 75 \%$, age $\geq 50$ years and $\mathrm{BMI} \leq 23 \mathrm{~kg} / \mathrm{m}^{2}$ ); the patient profile with the lowest probability of isolation of NTM $(0 \%)$ in our series comprised FVC $<75 \%$ predicted and age $<50$ years.

\section{Discussion}

Our study indicates that the prevalence of NTM isolates is high in patients with non-CF bronchiectasis and that $M$. avium complex is the most commonly isolated
Table 1 Patient characteristics for patients with and without Non-tuberculous mycobacteria (NTM) cultured from their sputum during the study period

\begin{tabular}{|c|c|c|c|}
\hline \multirow[t]{2}{*}{ Variable } & $\begin{array}{l}\text { NTM } \\
\text { positive }\end{array}$ & $\begin{array}{l}\text { NTM } \\
\text { negative }\end{array}$ & \multirow[t]{2}{*}{$p$-value } \\
\hline & $(n=18)$ & $(n=200)$ & \\
\hline Age, $y r^{a}$ & $64(13.3)$ & $54.9(15.9)$ & 0.02 \\
\hline Age $\geq 50$ years & $\begin{array}{l}15 \\
(83.3 \%)\end{array}$ & $126(63 \%)$ & 0.06 \\
\hline Gender (\% females) $)^{b}$ & $78 \%$ & $60 \%$ & 0.2 \\
\hline $\mathrm{BMI}, \mathrm{kg} / \mathrm{m}^{2 \mathrm{a}}$ & $23.5(4.8)$ & $24.9(4.6)$ & 0.24 \\
\hline $\mathrm{BMI} \leq 23 \mathrm{~kg} / \mathrm{m}^{2 \mathrm{~b}}$ & $\begin{array}{l}11 \\
(61.1 \%)\end{array}$ & $65(32.5 \%)$ & 0.034 \\
\hline Idiopathic & $5(27.8 \%)$ & $60(30 \%)$ & 0.79 \\
\hline Post-infection & $6(33.3 \%)$ & $72(36 \%)$ & \\
\hline Systemic diseases & $4(22.2 \%)$ & $24(12 \%)$ & \\
\hline Immunodeficiency & 1 (5.6 \%) & $4(2 \%)$ & \\
\hline COPD & $2(11.1 \%)$ & $28(14 \%)$ & \\
\hline Ciliary dyskinesia & 0 & 7 (3.3\%) & \\
\hline Other & 0 & $5(2.5 \%)$ & \\
\hline Smoking history (pack-years) ${ }^{c}$ & $22.2(33)$ & $11.6(25.6)$ & 0.2 \\
\hline Dyspnea (mMRC) & $1.29(1.3)$ & $1.33(1.13)$ & 0.8 \\
\hline $\begin{array}{l}\text { Macroscopic appearance of sputum } \\
\text { (muco-purulent or purulent) }^{\mathrm{a}}\end{array}$ & 7 (39\%) & $\begin{array}{l}125 \\
(62.5 \%)\end{array}$ & 0.045 \\
\hline Cystic bronchiectasis $^{\mathrm{b}}$ & $4(22 \%)$ & $52(26 \%)$ & 0.7 \\
\hline Number of affected lobes ${ }^{a}$ & $2.9(1.3)$ & $2.6(1.2)$ & 0.2 \\
\hline FVC, $\%$ predicted $^{a}$ & $82.5(23)$ & $73.5(24)$ & 0.09 \\
\hline FVC $\geq 75 \%$ predicted $^{b}$ & $\begin{array}{l}14 \\
(77.8 \%)\end{array}$ & $94(47 \%)$ & 0.011 \\
\hline $\mathrm{FEV}_{1}, \%$ predicted $^{\mathrm{a}}$ & $72.3(26)$ & $63(25)$ & 0.15 \\
\hline Chronic $P$. aeruginosa infection ${ }^{b}$ & $5(28 \%)$ & $88(44 \%)$ & 0.1 \\
\hline Chronic $H$. influenzae infection ${ }^{b}$ & $2(11 \%)$ & $46(23 \%)$ & 0.1 \\
\hline $\begin{array}{l}\text { Chronic bacterial infection, other } \\
\text { PPMs }^{\text {b }}\end{array}$ & $2(12 \%)$ & $90(45 \%)$ & 0.05 \\
\hline Isolation of Aspergillus spp ${ }^{\mathrm{b}}$ & $7(39 \%)$ & $52(26 \%)$ & 0.2 \\
\hline Macrolides (chronic use) ${ }^{\mathrm{b}}$ & $3(17 \%)$ & $22(11 \%)$ & 0.4 \\
\hline Antibiotics (chronic use) ${ }^{b}$ & $4(22 \%)$ & $70(35 \%)$ & 0.2 \\
\hline Systemic steroids (chronic use) & $1(6 \%)$ & $8(4 \%)$ & 0.6 \\
\hline Hospital admissions ${ }^{c}$ & $0.5(0.7)$ & $0.7(1.1)$ & 0.5 \\
\hline Exacerbations $^{c}$ & $2.18(1.7)$ & $3(2.1)$ & 0.2 \\
\hline
\end{tabular}

$B M I$ body mass index, COPD chronic obstructive lung disease, $m M R C$ modified Medical Research Council, PPMs potentially pathogenic microorganisms aExpressed as mean (SD)

${ }^{b}$ Expressed as frequency, number (\%)

'Expressed as median (IQR)

microorganism. Based on our results, $\mathrm{FVC} \geq 75 \%$ predicted, age $\geq 50$ years, and $\mathrm{BMI} \leq 23 \mathrm{~kg} / \mathrm{m}^{2}$ were independently associated with the presence of NTM at least once in patients with non-CF bronchiectasis. Furthermore, these patients tended to be less frequently chronically infected by $P$. aeruginosa and $H$. influenzae. 
Table 2 Logistic regression showing the factors independently associated with the presence of Non-tuberculous mycobacteria in the sputum of patients with non-cystic fibrosis bronchiectasis

\begin{tabular}{lllll}
\hline Variable & B & OR & $95 \% \mathrm{IC}$ & $p$ \\
\hline FVC $\geq 75 \%$ predicted & 1.58 & 4.84 & $1.47-15.9$ & 0.009 \\
Age $\geq 50$ years & 1.56 & 4.74 & $1.25-17.97$ & 0.022 \\
BMI $\leq 23 \mathrm{~kg} / \mathrm{m}^{2}$ & 1.09 & 2.97 & $1.03-8.58$ & 0.045 \\
\hline
\end{tabular}

Full adjusted model include: $\mathrm{FVC} \geq 75 \%$ predicted, age $\geq 50$ years, body mass index $(\mathrm{BMI}) \leq 23 \mathrm{~kg} / \mathrm{m}^{2}$, presence of chronic bacterial infection by potentially pathogenic microorganisms and macroscopic appearance of the sputum (mucous vs mucopurulent or purulent)

In the present study, the prevalence of NTM was $8.3 \%$, which is consistent with values reported previously in adult patients with non-CF bronchiectasis (2 to $37 \%)$ [15-17]. Consistent with previous findings [7], $M$. avium complex was the most frequently isolated pathogen. Again, as indicated by other authors $[15,16]$, it is notable that smears were positive in only $33 \%$ of patients with positive cultures.

Data on the natural history, prognosis, and associated factors of adult patients with non-CF bronchiectasis and coexisting NTM infection are limited, and most studies were conducted in CF patients with bronchiectasis. In both CF and non-CF patients, age is the only risk factor clearly associated with NTM [17-19]. However, in the present study, we found that other factors were associated with isolation of NTM. In this sense, NTM-positive patients tended to have better lung function, lower BMI, lower frequency of chronic $P$. aeruginosa infection, and a higher frequency of Aspergillus. NTM-positive patients also had fewer exacerbations and less frequently took chronic antibiotic therapy. A previous report found that NTM were more likely to be isolated in female patients with low BMI [17].

We found that age $\geq 50$ years and FVC $\geq 75 \%$ predicted were independently associated with isolation of
NTM. The association between NTM and significantly older age in patients with mild disease may be a phenomenon of repeated, prolonged environmental exposure that is not related to the severity of pulmonary disease. Olivier et al. suggest that patients with more severe lung disease could die before having sufficient exposure time to acquire and retain mycobacteria [6].

Although several published reports on CF and non-CF bronchiectasis patients found that lung function and colonization by $P$. aeruginosa were positively, negatively, or inconsequentially associated with the development of NTM [15-17, 20-23], a large American multicenter study in CF patients showed that patients who were culture-positive for NTM were older, had milder disease, and were more likely to be colonized with Staphylococcus aureus but not with $P$. aeruginosa [6]. In the present study, NTM-positive patients tended to have better lung function and a lower frequency of chronic $P$. aeruginosa infection. Aspergillus was also more frequently isolated in this group. In fact, the factor most associated with the presence of bronchiectasis was mild functional impairment (FVC $\geq 75 \%$ predicted). Patients with mild functional impairment presented a greater prevalence of NTM (13\%), making this variable the best predictor of isolation of NTM. Given that P. aeruginosa infection has been associated with worse pulmonary function and poor prognosis [24, 25], NTM-positive patients in our study, who were less commonly infected by $P$. aeruginosa, should have less impaired pulmonary function and a longer life expectancy. However, we cannot rule out the possibility that this association results from the difficulty in recovering mycobacteria from sputum with $P$. aeruginosa despite decontamination procedures $[6,26]$.

Although no clear association between NTM and Aspergillus spp has been demonstrated, several investigators have reported that NTM infection is associated with Aspergillusrelated diseases in patients with non-CF bronchiectasis [18]

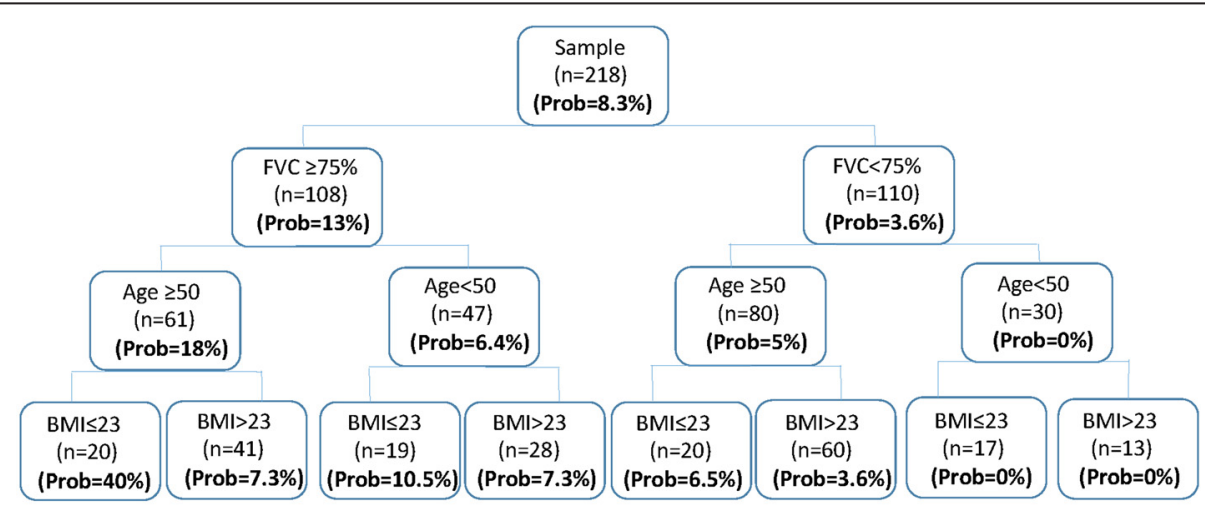

Prob = probability BMI = body mass index

Fig. 2 Probability of the isolation of non-tuberculous mycobacteria in patients with non-cystic fibrosis bronchiectasis by patients characteristics. Prob = probability; $\mathrm{BMI}=$ body mass index 
and with higher rates of $A$. fumigatus culture in patients with CF $[21,23,27]$ than in NTM-negative patients. Given that airway colonization by filamentous fungi has been associated with older age in CF patients with bronchiectasis [28, 29], perhaps the more frequent isolation of Aspergillus could be explained by the older age of NTM-positive patients in our study.

One of the key strengths of the present study is the very large cohort of patients with multiple sputum samples during the 5-year follow-up and the fact that we were able to construct an individual probability tree for the presence of bronchiectasis depending on the characteristics of the patient. Also noteworthy is the association with better pulmonary function and a trend towards an association with lower frequency of chronic infection by $P$. aeruginosa. Furthermore, in other studies on bronchiectasis and NTM, the study population was entered from a single tertiary referral center, while ours is the only multicenter study with a large patient population.

In addition to the logical restraints inherent to a retrospective design, our study is limited by the fact that the only factors we found to be clearly associated with isolation of NTM were age, lung function, and BMI. This finding may have been due to underpowering, since only 18 patients were NTM-positive. Furthermore, since we did not perform bronchoscopy, we could have underestimated the prevalence of NTM.

\section{Conclusions}

In conclusion, we report an $8.3 \%$ prevalence of NTM (2.3\% meeting ATS/IDS microbiological criteria for infection) in the sputum of screened non-CF patients with bronchiectasis in specialist clinics. M. avium complex appears to be the most common pulmonary NTM in this group of patients. Patients who are culture-positive for NTM are older and have a lower BMI and milder disease in functional terms. They also tend to have less frequent chronic infection by $P$. aeruginosa and more frequent isolation of $A$. fumigatus than culture-negative patients. Larger sample sizes will enable us to better evaluate the extent of the association of bronchiectasis with NTM, assess the clinical impact of the disease, and identify factors that are clearly associated with NTM in non-CF patients with bronchiectasis.

\section{Acknowledgments}

We thank the microbiologists in the participating hospitals (Enrique Gómez Mampaso, Diego Domingo and Pilar Blanch) for their help in microbiological studies. We also thank Gerad Muñoz for acquisition and analysis of data.

\section{Funding}

Funding for the study (including the article-processing charge) was received from Praxis Pharmaceutical Praxis Pharmaceutical and Zambon Laboratories (Zambon Laboratories). This study is included in the Programas Integrados de Investigación (PII) of Bronchiectasis of SEPAR (Spanish Society of Pulmonology and Thoracic Surgery).
Availability of data and materials

The datasets supporting the conclusions of this article are included within the article.

\section{Authors' contributions}

LM and MAM contributed to the conception and design of the study, acquisition of data, and analysis and interpretation of data. They also drafted the article and critically reviewed it for important intellectual content. Both authors approved the final version. RG contributed to the acquisition analysis, and interpretation of data. She also helped draft the article and critically reviewed it for important intellectual content. She approved the final version. CO contributed to the acquisition, analysis, and interpretation of data. She also helped draft the article and critically reviewed it for important intellectual content. She approved the final version. MV contributed to the acquisition, analysis, and interpretation of data. She also helped draft the article and critically reviewed it for important intellectual content. She approved the final version. RN contributed to the acquisition, analysis, and interpretation of data. She also helped draft the article and critically reviewed it for important intellectual content. She approved the final version.

\section{Competing interests}

The authors declare that they have no competing interests.

\section{Consent for publication}

Not applicable.

\section{Ethics approval and consent to participate}

The study was approved by the Ethics and Research Committees of Hospital Ramón y Cajal (Madrid, Spain), Hospital Universitario de La Princesa (Madrid, Spain), Hospital Universitario Regional de Málaga (Málaga, Spain), Hospital Josep Trueta (Girona, Spain), Hospital Universitario y Politécnico La Fe (Valencia, Spain). Registration number of the coordinating center: 0088-89-2011).

\section{Author details}

${ }^{1}$ Pneumology Service, Chronic Bronchial Infection, Cystic Fibrosis and Bronchiectasis Unit, Hospital Universitario Ramón y Cajal, Carretera de Colmenar Km 9,100, 28034 Madrid, Spain. ${ }^{2}$ Pneumology Service, Hospital La Princesa, Institute for Health Research (IP), Hospital Universitario de La Princesa, Madrid, Spain. ${ }^{3}$ Pneumology Service, Hospital Universitario Regional de Málaga, Instituto de Biomedicina de Málaga (IBIMA), Málaga University, Málaga, Spain. ${ }^{4}$ Bronchiectasis Group (Girona Biomedical Research Institute) IDIBGI, Dr Trueta University Hospital, Girona, Spain; CIBER de Enfermedades Respiratorias (Ciberes CB06/06/0030), Instituto de Salud Carlos III, Madrid, Spain. ${ }^{5}$ Pneumology Service, Hospital Universitario y Politécnico La Fe, CIBERes, CIBER de Enfermedades Respiratorias, Valencia, Spain.

Received: 21 June 2015 Accepted: 10 August 2016

Published online: 22 August 2016

\section{References}

1. Cole P. The damaging role of bacteria in chronic lung infection. J Antimicrob Chemother. 1997;40 Suppl A:5-10.

2. Nicotra MB, Rivera M, Dale AM, Shepherd R, Carter R. Clinical, pathophysiologic, and microbiologic characterization of bronchiectasis in an aging cohort. Chest. 1995;108:955-61.

3. Falkinham 3rd JO. Mycobacterial aerosols and respiratory disease. Emerg Infect Dis. 2003:9:763-7.

4. Marras TK, Chedore P, Ying AM, Jamieson F. Isolation prevalence of pulmonary non-tuberculous mycobacteria in Ontario, 1997-2003. Thorax. 2007;62:661-6.

5. Tanaka E, Amitani R, Niimi A, Suzuki K, Murayama T, Kuze F. Yield of computed tomography and bronchoscopy for the diagnosis of Mycobacterium avium complex pulmonary disease. Am J Respir Crit Care Med. 1997:155:2041-6.

6. Olivier KN, Weber DJ, Wallace Jr RJ, et al. Nontuberculous mycobacteria. I: multicenter prevalence study in cystic fibrosis. Am J Respir Crit Care Med. 2003; 167:828-34.

7. Chu $H$, Zhao $L$, Xiao $H$, et al. Prevalence of nontuberculous mycobacteria in patients with bronchiectasis: a meta-analysis. Arch Med Sci. 2014;10:661-8. 
8. Vendrell M, de Gracia J, Olveira C, et al. Diagnosis and treatment of bronchiectasis. Spanish Society of Pneumology and Thoracic Surgery. Arch Bronconeumol. 2008:44:629-40.

9. De Boeck K, Wilschanski M, Castellani C, et al. Cystic fibrosis: terminology and diagnostic algorithms. Thorax. 2006;61:627-35.

10. Naidich DP, McCauley DI, Khouri NF, Stitik FP, Siegelman SS. Computed tomography of bronchiectasis. J Comput Assist Tomogr. 1982;6:437-44.

11. Gaga M, Bentley AM, Humbert M, et al. Increases in CD4+ T lymphocytes, macrophages, neutrophils and interleukin 8 positive cells in the airways of patients with bronchiectasis. Thorax. 1998;53:685-91.

12. Angrill J, Agustí C, Torres A. Bronchiectasis. Curr Opin Infect Dis. 2001;14:193-7.

13. Lee TW, Brownlee KG, Conway SP, Denton M, Littlewood JM. Evaluation of a new definition for chronic Pseudomonas aeruginosa infection in cystic fibrosis patients. J Cyst Fibros. 2003;2:29-34

14. Griffith DE, Aksamit T, Brown-Elliott BA, ATS Mycobacterial Diseases Subcommittee, American Thoracic Society, Infectious Disease Society of America, et al. An official ATS/IDSA statement: diagnosis, treatment, and prevention of nontuberculous mycobacterial diseases. Am J Respir Crit Care Med. 2007;175:367-416.

15. Wickremasinghe $M$, Ozerovitch $L$, Davies $G$, et al. Nontuberculous mycobacteria in patients with bronchiectasis. Thorax. 2005;60:1045-51.

16. Fowler SJ, French J, Screaton NJ, et al. Nontuberculous mycobacteria in bronchiectasis: Prevalence and patient characteristics. Eur Respir J. 2006; 28:1204-10.

17. Mirsaeidi M, Hadid W, Ericsoussi B, Rodgers D, Sadikot RT. Non-tuberculous mycobacterial disease is common in patients with non-cystic fibrosis bronchiectasis. Int J Infect Dis. 2013;17:e1000-4.

18. Kunst H, Wickremasinghe M, Wells A, Wilson R. Nontuberculous mycobacterial disease and Aspergillus-related lung disease in bronchiectasis. Eur Respir J. 2006;28:352-7.

19. Winthrop KL, McNelley E, Kendall B, et al. Pulmonary nontuberculous mycobacterial disease prevalence and clinical features: an emerging public health disease. Am J Respir Crit Care Med. 2010;182:977-82.

20. Girón RM, Máiz L, Barrio I, Martínez MT, Salcedo A, Prados C. Nontuberculous mycobacterial infection in patients with cystic fibrosis: a multicenter prevalence study. Arch Bronconeumol. 2008;44:679-84

21. Levy I, Grisaru-Soen G, Lerner-Geva L, et al. Multicenter cross-sectional study of nontuberculous mycobacterial infections among cystic fibrosis patients, Israel. Emerg Infect Dis. 2008;14:378-84.

22. Radhakrishnan DK, Yau Y, Corey M, et al. Non-tuberculous mycobacteria in children with cystic fibrosis: isolation, prevalence, and predictors. Pediatr Pulmonol. 2009;44:1100-6.

23. Esther Jr CR, Esserman DA, Gilligan P, Kerr A, Noone PG. Chronic Mycobacterium abscessus infection and lung function decline in cystic fibrosis. J Cyst Fibros. 2010;9:117-23.

24. Evans SA, Turner SM, Bosch BJ, Hardy CC, Woodhead MA. Lung function in bronchiectasis: the influence of Pseudomonas aeruginosa. Eur Respir J. 1996;9:1601-4.

25. Martínez-García MA, de Gracia J, Vendrell Relat M, et al. Multidimensional approach to non-cystic fibrosis bronchiectasis. The FACED score. Eur Respir J. 2014;43:1357-67

26. Oliver A, Máiz L, Cantón R, Escobar H, Baquero F, Gómez-Mampaso E. Nontuberculous mycobacteria in patients with cystic fibrosis. Clin Infect Dis. 2001:32:1298-303.

27. Paugam A, Baixench MT, Demazes-Dufeu N, et al. Characteristics and consequences of airway colonization by filamentous fungi in 201 adult patients with cystic fibrosis in France. Med Mycol. 2010;48 Suppl 1:S32-6.

28. Máiz L, Cuevas M, Lamas A, Sousa A, Quirce S, Suárez L. Aspergillus fumigatus and Candida albicans in cystic fibrosis: clinical significance and specific immune response involving serum immunoglobulins $G, A$, and $M$. Arch Bronconeumol. 2008:44:146-51.

29. Valenza G, Tappe D, Turnwald D, et al. Prevalence and antimicrobial susceptibility of microorganisms isolated from sputa of patients with cystic fibrosis. J Cyst Fibros. 2008:7:123-7.

\section{Submit your next manuscript to BioMed Central and we will help you at every step:}

- We accept pre-submission inquiries

- Our selector tool helps you to find the most relevant journal

- We provide round the clock customer support

- Convenient online submission

- Thorough peer review

- Inclusion in PubMed and all major indexing services

- Maximum visibility for your research

Submit your manuscript at www.biomedcentral.com/submit

) Biomed Central 